
\title{
health in Britain
}

Largest national survey offers insight into sexual

$\mathrm{T}$ he third British National Survey of Sexual Attitudes and Lifestyles (Natsal-3) - one of the largest ever studies of sexual health and behaviour in a single country-has been reported in The Lancet. This large stratified probability sample survey (of data collected from 15,162 adults aged $16-74$ years living in Britain between September 2010 and August 2012) provides information that could be used to improve sexual health in the future. Natsal-3 follows on from Natsal-1 (1990-1991) and Natsal-2 (1999-2001), offering unique insight into the changes in sexual health that have occurred in Britain over the past 25 years.

Since Natsal-2 was performed, a number of strategies have been implemented across Britain to improve sexual health in relation to infection, including the National Strategy for Sexual Health and HIV in England (2001) and the English National Chlamydia Screening Programme (NCSP; 2004), with corresponding initiatives in Scotland and Wales. Urine samples from 4,550 Natsal-3 participants aged 16-44 years who had at least one sexual partner over their lifetime, were tested for chlamydia, gonorrhoea, high-risk human papillomavirus (hrHPV) and HIV.

Chlamydia and hrHPV were the most commonly detected infections. Chlamydia was detected in $\sim 1 \%$ of the overall sample population, with higher rates in younger adults (aged $16-24$ years; $3.1 \%$ and $2.3 \%$ in women and men, respectively). Overall prevalence of $\mathrm{hrHPV}$ was $15.9 \%$ in women and $8.4 \%$ in men, rates which are similar to those reported in Natsal-2. Most people infected with chlamydia or hrHPV were low-risk individuals with few partners, suggesting that broad population-wide prevention initiatives are needed for both infections.

By contrast, HIV and gonorrhoea were relatively uncommon in Natsal-3. Gonorrhea was reported in $<0.1 \%$ of participants, all of whom had known risk

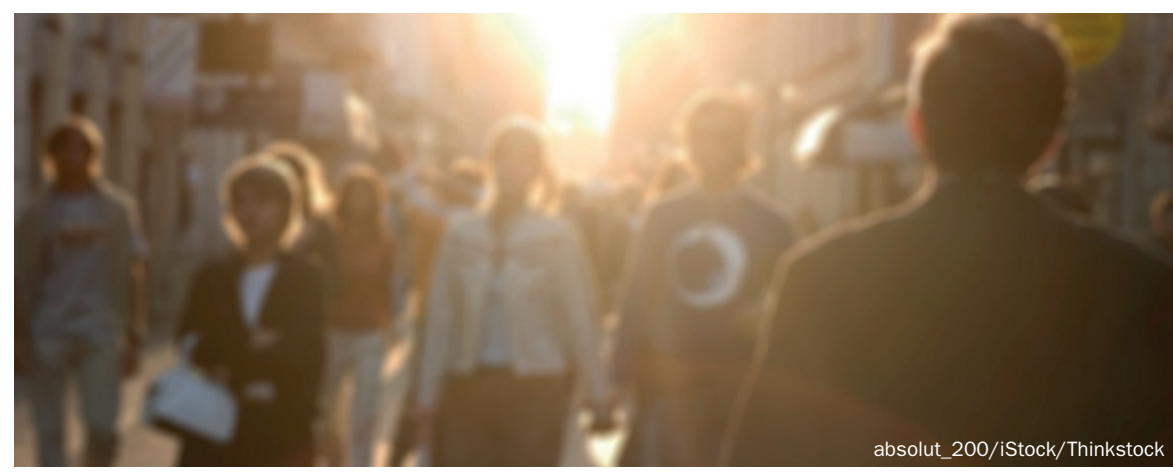

factors for the infection (aged 20-24 years and reported sex with at least two partners in the past year without use of condoms). Similarly, the $0.1 \%$ of women and $0.2 \%$ of men who tested positive for HIV were considered high risk. The investigators suggest that targeted interventions are needed for these individuals.

Overall, rates of attendance at sexual health clinics were much higher in Natsal-3 than in previous surveys, providing some reassurance that sexual health strategies are working. However, while sexually transmitted infections continue to be reported, the need for promotion of these strategies remains.

Another goal of Natsal-3 was to highlight the importance of sexual function as an indicator of sexual health and to collect information on the status of sexual function in Britain. To do so, the investigators constructed a novel validated tool, Natsal-SF, which is suitable for measuring sexual function in both men and women, and emphasizes the importance of relationship context; the investigators wanted to avoid a focus on clinical pathology and the medicalization of sexual function.

4,913 men and 6,777 women who were sexually active in the past year completed Natsal-SF. $51 \%$ of women and $42 \%$ of men reported having sexual difficulties (including lack of interest, anxiety, pain, lack of climax, early climax and arousal difficulties) lasting $\geq 3$ months over the preceding year. Using the lowest quintile of Natsal-SF scores to define low sexual function, associations were found with depression, unemployment, self-reported health status and age, although notably the age effect was not observed beyond the 55-64-year-old age group. Low sexual function was not uncommon in young people (16.4\% in those aged $25-34$ years, $20.4 \%$ in the $35-44$-year-old age group), indicating that higher provision of advice might be needed to improve their sex lives.

A separate Natsal-3 study specifically investigated the link between poor general health and sexual function (measured as recent sexual activity, sexual satisfaction and sexual response problems [vaginal dryness and erectile difficulties]). $16.6 \%$ of men and $17.2 \%$ of women reported that their health had affected their sex life in the past year, with approximately $60 \%$ of those in bad health claiming the same.

The wealth of data in this highprofile survey is hoped to lead to wider acceptance of sexual health as a crucial indicator of overall health and encourage its inclusion in public health policy.

\section{Sarah Payton}

